\title{
Prevalence and Clinical Correlation of Vitamin D Inadequacy in Traumatic Hip Fracture among Elderly Patients
}

\section{Tutaworn T, Phruetthiphat $0 *$ and Songpatanasilp T \\ Department of Orthopaedics, Phramongkutklao Hospital, Bangkok, Thailand}

*Corresponding author: Ong-art Phruetthiphat, Department of Orthopaedics, Phramongkutklao Hospital, Bangkok, Thailand

\section{Research Article \\ Volume 3 Issue 4}

Received Date: October 21, 2019

Published Date: December 16, 2019

DOI: $10.23880 /$ jobd-16000191

\section{Abstract}

Introduction: High prevalence of vitamin D deficiency has been described in more than 1 billion people around the world, especially in Middle-East and Asia. In Thailand, the largest study of vitamin D status in normal population $(\mathrm{N}=2641)$ identified overall 45.2 percent of vitamin $\mathrm{D}$ inadequacy in normal population. However, there was no study focusing on Vitamin D inadequacy in traumatic elderly patients. The purpose of this study is going to define the prevalence of vitamin D inadequacy in traumatic hip fracture among elderly patients and to identify a clinical correlation.

Materials and Methods: A prospective study of elderly patients (more than 60 years old) with low energy trauma who underwent orthopaedic surgery at Phramongkutklao Hospital between the period of January 2016 and December 2016. All hip fracture patients underwent orthopedic surgery were collected for demographic data, comorbidities including Charlson Comorbidity Index. Primary outcome was to identify a prevalence of vitamin D inadequacy $(<30 \mathrm{ng} / \mathrm{ml})$ and vitamin D deficiency $(<20 \mathrm{ng} / \mathrm{ml})$ in hip fracture underwent orthopedic surgery. Secondary outcome was to define association of demographic data, comorbidity with vitamin D level. Additionally, comparison the ambulatory status and mechanical failure between normal vitamin D and vitamin D inadequacy

Results: The serum 25(OH)D levels for this study was normally distributed, with a mean of $25.0 \pm 12.2 \mathrm{ng} / \mathrm{ml} \mathrm{in}$ all patients, Prevalence of vitamin D inadequacy was 70.5 percent $(n=103)$ and the prevalence of vitamin D deficiency was 37.7 percent $(n=55)$. There was no significant difference of ambulatory status between vitamin $D$ inadequacy and sufficient vitamin D groups ( $\mathrm{p}=0.958)$. Additionally, there was not different in mechanical failure between groups $(4.5 \%$ and $0 \%, \mathrm{p}=1.000$ ).

Conclusion: Vitamin D inadequacy is so common in traumatic elderly patients. We should raise attentiveness among physician to determine vitamin D status. Public health strategy should add knowledge about diet, supplements or adequate sun exposure to elderly population. Vitamin D inadequacy did not affect the outcome including time to union and ambulatory status at 1 year follow up.

Keywords: Vitamin D; Fractures; Patients 


\section{Journal of Orthopedics \& Bone Disorders}

\section{Introduction}

Vitamin D plays a vital role not only in making our bones strong and it is important in bone mineralization and maintenance of bone quality through the regulation of calcium and skeletal homeostasis, but also it maintain our immune system, reproductive system, musculoskeletal system and integumentary system in all ages and races [1]. The minimum level of $30 \mathrm{ng} / \mathrm{ml}$ is necessary in older adults to minimize the risk of fractures due to falls [2,3]. High prevalence of vitamin D deficiency has been described in more than 1 billion people around the world, especially in Middle-East and Asia [4] and it has been seen in all races, age groups and ethnic background [2]. There are a few data demonstrating a screening for vitamin D deficiency in asymptomatic adults or during pregnancy $[5,6]$ while most experts agree that it is not necessary to perform broad screening of serum $25(\mathrm{OH}) \mathrm{D}$ levels in the general population or during pregnancy [5,7].

Systematic review and meta-analysis identified prevalence of Vitamin D inadequacy in traumatic patient, they found that $70 \%$ of all fracture patients and $73 \%$ of fragility fracture (age more than 50 years old) have Vitamin D level less than $30 \mathrm{ng} / \mathrm{ml}^{4}$. In Thailand, there are several studies demonstrating the prevalence of vitamin D deficiency. Soontrapa S. et al. [8] found that 66.3 percent of elderly Thai women in Khonkaen province have Vitamin D deficiency [25(OH)D level $<35 \mathrm{ng} / \mathrm{ml}$ ] while the largest study of vitamin D status in normal population $(\mathrm{N}=2641)$ identified overall 45.2 percent of vitamin D inadequacy in normal population [9]. However, there was no study focusing on Vitamin D inadequacy in traumatic elderly patients. So, the purpose of this study is going to define the prevalence vitamin $\mathrm{D}$ inadequacy in traumatic hip fracture among elderly patients.

\section{Materials and Methods}

\section{Subjects}

We performed a prospective study of hip fracture among elderly patients (more than 60 years old) with low energy trauma who underwent orthopaedic surgery at Phramongkutklao Hospital between the period of January 2016 and December 2016. After approval by our institutional review board and got informed consent from patients/first degree relatives, study subjects needed to obtain serum 25(OH)D at the time of admission, prior to surgery. Patients with high risk for Vitamin D inadequacy (Serum creatinine more than $2 \mathrm{md} / \mathrm{dL}$, mal absorption syndrome, post gastrectomy, active liver disease, acute myocardial infarction, anorexia nervosa and steroid dependency), pathological fracture and multiple sites of fracture were excluded. Finally, 146 elderly patients were recruited in this study. We also further randomly investigated a serum 25(OH) vitamin D level in 146 normal elderly populations without any fracture for the control group.

\section{Laboratory Studies}

Serum Calcium, phosphorus, and albumin levels were measured with use of standard automated laboratory method at our hospital. Vitamin D level was identified by using "chemiluminescent immunoassay" on Roche property Asset No. 9006102 model cobase 601 for detect total serum 25-hydroxyvitamin D. This machine can interpret level $3.00-70.00 \mathrm{ng} / \mathrm{ml}$ (if less than $3 \mathrm{ng} / \mathrm{ml}$, it's interpret $3 \mathrm{ng} / \mathrm{ml}$ and if more than $70 \mathrm{ng} / \mathrm{ml}$, it's interpret $70 \mathrm{ng} / \mathrm{ml}$ ). Vogeser et al. compared reliability of this machine with gold standard (liquid Chomatography Tandem Mass Spectrometry) and they found that this machine was appropriate for hospital [10].

\section{Background Data}

Patients demographic and background that may be the risk factors for Vitamin D inadequacy were collected by researcher. Variables included age, sex, height and body weight for calculate body-mass index (BMI), location of fracture, ambulatory status before fracture, history of previous fracture, timing of sun exposure per day, timing from fracture to surgery and comorbidities for calculate Charlson Comorbidity Index [11]. Age was further categorized into three groups (sixty to sixty-nine, seventy to seventy-nine and more than 80 years) to investigate their potential effects on serum Vitamin D level. Bodymass index was grouped into two groups: less than 30 $\mathrm{kg} / \mathrm{m}^{2}$ and equal or above $30 \mathrm{~kg} / \mathrm{m}^{2}$.

\section{Outcome Measures}

Our primary objective was prevalence of vitamin D inadequacy (less than $30 \mathrm{ng} / \mathrm{ml}$ ) and vitamin D deficiency (less than $20 \mathrm{ng} / \mathrm{ml}$ ) in traumatic hip fracture among elderly patients.

Secondary objectives were association of demographic data (age, gender, BMI) and vitamin D level. We also identified an association between Charlson Comorbidity Index (CCI), preoperative albumin, ambulatory status before fracture, history of previous fracture, timing of sun exposure per day, timing from fracture to surgery, length 


\section{Journal of Orthopedics \& Bone Disorders}

of hospital stays with vitamin D level. We also followed patients for 1 year to find an association between ambulatory status at 1 year, time to union and failure of implants with vitamin D level (sufficiency and inadequacy groups).

\section{Statistical Analysis}

Primary outcome was analyzed by prevalence (number and percentage) of Vitamin D inadequacy (less than $30 \mathrm{ng} / \mathrm{ml}$ ) and deficiency (less than $20 \mathrm{ng} / \mathrm{ml}$ ) in overall population.

In secondary outcomes, univariate and multivariate logistic regression models were used to assess the independently relative risk for vitamin-D inadequacy in each potential risk factor (age group, sex, body-mass index, preoperative albumin, ambulatory status before fracture, history of previous fracture and timing of sun exposure per day, timing from fracture to surgery, length of hospital stays).

Independent t-test was used for Charlson Comorbidity Index while chi-square test was applied for preoperative albumin, history of previous fracture and timing from fracture to surgery. Ambulatory status before fracture, ambulatory status at 1 year, and failure of implant were analyzed by Fisher's exact test was used. The association of sun exposure per day, length of hospital stays, Charlson Comorbidity Index, time to union and vitamin inadequacy was analyzed by Mann-Whitney $U$ test. All parameters were performed with use of two-tailed tests. The level of significance was set at $\mathrm{p}$-value less than 0.05 .

\section{Results}

\section{Baseline Characteristics}

A total of 146 patients participated in this study, $24.7 \%(n=36)$ were men and $75.3 \%(n=110)$ were women (Table 1). Ages ranged from 60 to 100 years; with a mean age (and standard deviation) were $78.8 \pm 8.9$ years. The average body-mass index was $22.0 \pm 4.3 \mathrm{~kg} / \mathrm{m}^{2}$. Almost of our population $(97.9 \%, \mathrm{n}=143)$ was not obesity (bodymass index less than $30 \mathrm{~kg} / \mathrm{m}^{2}$ ). According to pre-fracture status, these patients can walk independently approximately $60 \%(n=88)$ and they had no previous history of fracture $(85.6 \%, \mathrm{n}=125)$.

\begin{tabular}{|c|c|c|c|}
\hline Characteristics & Male (n=36) & Female $(n=110)$ & Total $(n=146)$ \\
\hline Age ---yr & $78.0 \pm 8.2$ & $79.1 \pm 9.1$ & $78.8 \pm 8.9$ \\
\hline BMI ---kg/m2 & $23.0 \pm 4.6$ & $21.6 \pm 4.1$ & $22.0 \pm 4.3$ \\
\hline Vitamin D -- ng/ml & $25.6 \pm 12.6$ & $24.5 \pm 12.1$ & $25.0 \pm 12.2$ \\
\hline CCI --- total & $4.7 \pm 1.4$ & $4.8 \pm 1.5$ & $4.8 \pm 1.5$ \\
\hline Albumin ---g/dL & $3.7 \pm 0.4$ & $3.7 \pm 0.5$ & $3.7 \pm 0.5$ \\
\hline \multicolumn{4}{|c|}{ Previous fx --- no. (\%) } \\
\hline No & $31(86.1)$ & $94(85.5)$ & $125(85.6)$ \\
\hline Yes & $5(13.9)$ & $16(14.5)$ & $21(14.4)$ \\
\hline \multicolumn{4}{|c|}{ Pre-fracture status --- no. (\%) } \\
\hline Independent & $25(69.4)$ & $63(57.3)$ & $88(60.3)$ \\
\hline Gait aid & $11(30.6)$ & $45(40.9)$ & $56(38.3)$ \\
\hline
\end{tabular}

Table 1: Baseline characteristics of the study populations (divided by gender).

* Plus-minus values are means $\pm \mathrm{SD}$, fx means fracture CCI means Charlson Comorbidity Index

$\%=$ percentages of each parameter divided by each column

\section{Prevalence of Vitamin D Inadequacy}

The serum 25(OH)D levels for this study was normally distributed, with a mean of $25.0 \pm 12.2 \mathrm{ng} / \mathrm{ml}$ in all patients, $25.6 \pm 12.6 \mathrm{ng} / \mathrm{ml}$ in male, $24.5 \pm 12.1 \mathrm{ng} / \mathrm{ml}$ in female, and minimum and maximum values of 3.0 and $66.89 \mathrm{ng} / \mathrm{ml} \mathrm{(Table} \mathrm{1).} \mathrm{Prevalence} \mathrm{of} \mathrm{vitamin} \mathrm{D}$ inadequacy $(<30 \mathrm{ng} / \mathrm{ml})$ was 70.5 percent $(\mathrm{n}=103)$ (Table
2) and the prevalence of vitamin D deficiency $(<20 \mathrm{ng} / \mathrm{ml})$ was 37.7 percent $(n=55)$.

\section{Vitamin D Associated with History of Previous Fracture}

Those patients with previous history of fracture was not significantly higher rate of vitamin D inadequacy 


\section{Journal of Orthopedics \& Bone Disorders}

comparing to those without previous history of fracture ( $57.1 \%$ vs $42.9 \%$, $\mathrm{P}=0.168$, respectively)

\section{Vitamin D Associated with Timing of Sun Exposure and Time from Fracture to Surgery}

The average median time of sun exposure (Table 2) was 15 minutes a day (minimum, maximum $=0,4$ hours) in vitamin $\mathrm{D}$ inadequacy group while it was 30 minutes a day (minimum, maximum $=0,5$ hours) for sufficient vitamin D group, which were significantly different between both groups $(p<0.001)$.

Timing from fracture to surgery, the former group was significantly higher rate of waiting for surgery more than 1 week comparing to the latter group $(77.8 \%$ vs $22.2 \%$, $\mathrm{p}=0.013$ ). However, total length of hospital stay was not significantly different between both groups (mean 14 days, $\mathrm{p}$-value $=0.604$ ).

\begin{tabular}{|c|c|c|c|}
\hline Factors & Vit. $\mathrm{D}<30 \mathrm{ng} / \mathrm{ml}$ & Vit. $D \geq 30 \mathrm{ng} / \mathrm{ml}$ & P Value \\
\hline \multicolumn{3}{|c|}{ Age --- yr } & 0.042 \\
\hline Total --- no. (\%) & $103(70.5)$ & $43(29.5)$ & \\
\hline 60-69 ---no. (\%) & $22(88.0)$ & $3(12.0)$ & \\
\hline 70-79 ---no. (\%) & $31(64.6)$ & $17(35.4)$ & \\
\hline$\geq 80$--- no. (\%) & $50(69.9)$ & $23(30.1)$ & \\
\hline \multicolumn{3}{|c|}{ Gender } & 0.487 \\
\hline Male ---no. (\%) & $24(66.7)$ & $12(33.3)$ & \\
\hline Female ---no. (\%) & $79(71.8)$ & $31(28.2)$ & \\
\hline \multicolumn{3}{|c|}{ BMI -- kg/m2 } & $0.557 \dagger$ \\
\hline$<30$---no. (\%) & $100(69.9)$ & $43(30.1)$ & \\
\hline$\geq 30$---no. (\%) & $3(100.0)$ & $0(0)$ & \\
\hline CCI --- total & $4.75 \pm 1.76$ & $4.83 \pm 1.26$ & $0.736 ¥$ \\
\hline \multicolumn{3}{|c|}{ Albumin --- g/dL } & 0.164 \\
\hline$<3.5$---no. (\%) & $24(80.0)$ & $6(20.0)$ & \\
\hline$\geq 3.5$---no. (\%) & $79(68.1)$ & $37(31.9)$ & \\
\hline \multicolumn{3}{|c|}{ Previous $\mathrm{fx}$} & 0.168 \\
\hline No ---no. (\%) & $90(72.0)$ & $35(28.0)$ & \\
\hline Yes ---no. (\%) & $12(57.1)$ & $9(42.9)$ & \\
\hline \multicolumn{3}{|c|}{ Previous fx status } & $0.615 \dagger$ \\
\hline Independent ---no. (\%) & $60(68.2)$ & $28(31.8)$ & \\
\hline Gait aid ---no. (\%) & $41(73.2)$ & $15(26.8)$ & \\
\hline Bed ridden ---no. (\%) & $2(100.0)$ & $0(33.3)$ & \\
\hline $\begin{array}{c}\text { Timing of sun expose\# -- } \\
\text { hour/day }\end{array}$ & $0.25(0-5)$ & $0.5(0-4)$ & $<0.001 \ddagger$ \\
\hline \multicolumn{3}{|c|}{ Timing from fracture to surgery } & 0.013 \\
\hline$<7$ days --- no. $(\%)$ & $33(58.9)$ & $23(41.1)$ & \\
\hline$\geq 7$ days ---no. $(\%)$ & $70(77.8)$ & $20(22.2)$ & \\
\hline
\end{tabular}

Table 2: Baseline characteristics of the study populations (divided by Vitamin D status).

* Plus-minus values are means \pm SD., CCI means Charlson Comorbidity Index, †Fisher's exact test, ${ }^{¥}$ Independent t-test, Chisquare test, and ₹ Mann-Whitney U test for other P Value, \# Timing of sun exposure shows in median (minimummaximum), $\mathrm{P}$ value $<0.05$ considered statistical significance; $44 \%$ of all patients had sun exposure less than 0.25 hour/day

\section{Vitamin D Correlated with Ambulatory Status at 1 Year, Time to Union and Failure of Fixation}

We follow up this population for 1 year after fracture; eight patients were died while six patients were loss of follow-up. Finally, 132 patients were included for further analysis (Table 3). Even though most of them can ambulate by single cane $(72.0 \%, \mathrm{n}=95)$, only $20.0 \%$ $(n=19)$ can ambulate independently at one year follow up which was significantly different comparing to prefracture status $(60.3 \%, \mathrm{n}=88)$. 


\section{Journal of Orthopedics \& Bone Disorders}

Association of vitamin D and ambulatory status, there was no significant difference of ambulatory status between vitamin $D$ inadequacy and sufficient vitamin $D$ groups $(p=0.958)$. Generally, displaced femoral neck fracture subgroup treated by primary hemiarthroplasty cannot evaluate for bone union including clinical union and radiographic union while time to union was measured in subgroup of intertrochanteric fracture treated by proximal femoral nail anti-rotation (PFNA) fixation and they were further divided into clinical union and radiographic union. Mean time of clinical union and radiographic union were also not significantly different between both groups ( 4 weeks vs 6 weeks, $p=0.741$ and 10.5 weeks vs 10 weeks, p=0.566, respectively). Furthermore, in cases of fixation failure, we also focused only on intertrochanteric fracture (65 cases) that underwent PFNA fixation. Although two-third (67.7\%, $\mathrm{n}=44$ ) of this group had vitamin D inadequacy, only 2 cases had implant failure (PFNA blade cutout-both of them were in group of Vitamin D inadequacy). Additionally, there was comparable rate of fixation failure between vitamin $\mathrm{D}$ inadequacy and sufficient vitamin D groups $(4.5 \%$ vs $0 \%, \mathrm{p}=1.000)$.

\begin{tabular}{|c|c|c|c|}
\hline $\begin{array}{l}\text { Factors } \\
\end{array}$ & Vit. $\mathrm{D}<30 \mathrm{ng} / \mathrm{ml}$ & Vit. $D \geq 30 \mathrm{ng} / \mathrm{ml}$ & P Value \\
\hline Ambulatory status at 1 year & & & $0.958 \dagger$ \\
\hline Independent ---no. (\%) & $14(14.7 \%)$ & $5(13.5 \%)$ & \\
\hline Cane (single/tripod) ---no. (\%) & $69(72.6 \%)$ & $26(70.3 \%)$ & \\
\hline Walker ---no. (\%) & $10(10.6 \%)$ & $5(13.5 \%)$ & \\
\hline Bedridden ---no. (\%) & $2(2.1 \%)$ & $1(2.7 \%)$ & \\
\hline \multicolumn{4}{|c|}{ Time to union --- weeks } \\
\hline Clinical union & $4(2-30)$ & $6(3-18)$ & $0.741 \neq$ \\
\hline Radiographic union & $10.5(4-38)$ & $10(8-22)$ & $0.566 \ddagger$ \\
\hline \multicolumn{3}{|c|}{ Failure of implant fixation } & $1.000 \dagger$ \\
\hline No ---no. (\%) & $42(95.5 \%)$ & $21(100 \%)$ & \\
\hline Yes ---no. (\%) & $2(4.5 \%)$ & $0(0 \%)$ & \\
\hline
\end{tabular}

Table 3: Univariate analysis of ambulatory status at 1 year after fracture, time to union and failure of implant fixation according to Vitamin D status.

$\dagger$ Fisher's exact test, ₹ Mann-Whitney U test

$P$ value $<0.05$ considered statistical significance. $\%=$ percentages of each parameter divided by each column.

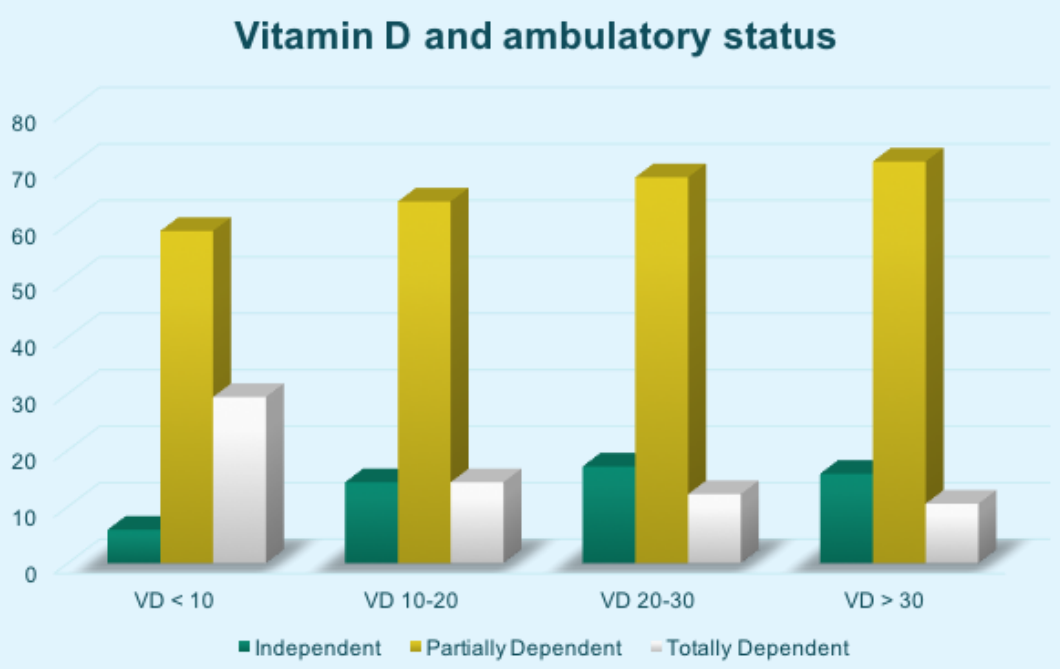

Table 4: Analysis of ambulatory status at one year follows up among each subgroup of vitamin D inadequacy and sufficient vitamin D level. 


\section{Journal of Orthopedics \& Bone Disorders}

Totally Dependent (wheel chair until bed ridden; white color box) was $29.4 \%, 14.3 \%, 12.2 \%$, and $10.5 \%$ for vitamin D level<10, 10-20, 20-30, and >30 ng/ml, respectively.

\section{Vitamin D in Normal Elderly Population}

One hundred and forty six vitamin D levels were randomly obtained and they were classified into 3 age groups (60-69 years, 70-79, and $\geq 80$ years). Mean serum vitamin D level was 31.4, 32.9, $34.8 \mathrm{ng} / \mathrm{ml}$, respectively. Mean serum Vitamin D level was higher in male than female in all age groups (Table 4).

\section{Discussion}

The purpose of this study is going to define the prevalence vitamin D inadequacy in traumatic hip fracture among elderly patients and to identify a clinical correlation of the vitamin D level. This study evaluated vitamin D status in traumatic elderly patients in Bangkok, Thailand, which is located in the central region of the country. The findings show that 70.5 percent have vitamin D inadequacy (less than $30 \mathrm{ng} / \mathrm{ml}$ ). This value is almost the same as Vitamin D status in traumatic elderly patients according to systematic review and metaanalysis by Sprague S, et al. [4] which $73 \%$ of fragility fracture (age more than 50 years old) had vitamin D level less than $30 \mathrm{ng} / \mathrm{ml}$. The prevalence of vitamin D inadequacy in our study was high that was comparable with Sprague's studies even though our patients live in tropical zone that should not have low vitamin D level. This may be explained by sedentary life style, almost elderly patients with hip fracture in our country are not only retired form work, but they also have low activity level preoperatively. Moreover, lack of vitamin D supplement in regular food especially in milk (only calcium supplement) and some patients (44 percentages) had sun exposure less than 0.25 hour.

Previous study by Chailurkit L, et al. [11] identified the largest vitamin D status in normal population in Thailand ( $n=2641$, age 14-98 years): overall population had 45.2 percentages of vitamin $\mathrm{D}$ inadequacy, particularly Bangkok is the highest rate of this problem (64.6\%). However, they have not demonstrated vitamin D status of traumatic hip fracture in elderly patients. Our study firstly identified the vitamin $\mathrm{D}$ level in hip fracture among elderly patients and we found a high prevalence of vitamin D deficiency in men that was compatible with previous study [12]. The association between gender and Vitamin D inadequacy was varied, some studies show higher percentage in women (very low serum 25(OH)D levels have been reported in the Middle East especially in women because of clothing habits while the others was common in men) [13]. However, the prevalence of vitamin $\mathrm{D}$ inadequacy was comparable between male and female in our study ( $66.7 \%$ vs $71.8 \%, \mathrm{p}=0.487)$.

Charlson Comorbidity Index (CCI) was high in hip fracture. Some data suggested that CCI more than 2 was higher risk for vitamin D inadequacy (Relative risk $=16.8$ ) [14]. The relationship between vitamin D inadequacy and obesity is still unknown, our study was not shown an association of them because we had low proportion (2.1 percent) of patients with obesity (BMI more than 30 $\left.\mathrm{kg} / \mathrm{m}^{2}\right)$.

Length of hospital stay in this study was not different between groups, agreed with Gumieiro DN, et al. [15] that serum Vitamin D level was not associated with length of hospital stay after hip fracture while Maier GS, et al. [16] found that length of stay in multivariate analysis was significantly higher rate associated with low Vitamin D levels ( $p$-value=0.002). However, the latter study collected the data from elective cases of hip or knee arthroplasty.

Not surprising, timing of sun exposure was strongly significant difference between two groups ( $p$-value less than 0.001). Timing from fracture to surgery was significantly longer in group with vitamin D less than 30 $\mathrm{ng} / \mathrm{ml}$ ( $\mathrm{p}$-value 0.013). Further analysis found that population in group of vitamin $\mathrm{D}$ inadequacy tend to have higher rate of anticoagulant usage (such as aspirin and clopidogrel) that had to stop before surgery, and they had higher rate of medical problem that needed to be stabilized before surgery.

When we followed up these population for 1 year, someone died, someone loss to follow up. Only 132 patients were completed follow up. Even though one year-ambulatory status was not different in both groups, almost of them dependently required gait aid for ambulation. Sixty-one percent of population can walk independently before fracture versus only twenty percent can walk independently after fracture at 1 year follow up. In the fixation group, union rate (clinical/radiographic union) was not significantly different. When focus on fixation with PFNA of hip legion, two cases of PFNA blade cutout was found, both of them occurred in group of vitamin $\mathrm{D}$ inadequacy. The association between fixation failure and vitamin D status was not found because of very low rate ( 2 out of 65 ) of fixation failure. The other 


\section{Journal of Orthopedics \& Bone Disorders}

reason why there was no different in fixation failure between groups because successful fixation mainly required proper surgical technique rather than vitamin $\mathrm{D}$ level.

Our study had several limitations. Firstly, a disproportionately large number of our patients were urbanization because most of them live in Bangkok. Given the predisposition of lower sun exposure when compared to population in rural area. Secondly, serum vitamin D levels were measured during various seasons. It is possible that seasonal variation may have affected the findings of this study. To solve this uncertainty, further study about individuals varies in their physiologic responses to sunlight and serum vitamin D levels in difference seasons should be done. As above-mentioned in limitations, so we need further studies to define the precise extent of vitamin D inadequacy in the orthopaedic surgery patient.

\section{Conclusion}

Vitamin D inadequacy is so common in traumatic hip fracture among elderly patients. We should raise attentiveness among physician to determine vitamin D status. Public health strategy should add knowledge about diet, supplements or adequate sun exposure to elderly population. Vitamin D inadequacy did not affect the outcome including time to union and ambulatory status at 1 year follow up.

\section{Source of Funding}

Maha Vajiralongkorn foundation funding support for this study.

\section{References}

1. ACOG Committee on Obstetric Practice (2011) ACOG Committee Opinion No. 495: Vitamin D: Screening and supplementation during pregnancy. Obstet Gynecol 118(1): 197-198.

2. Lips P (2001) Vitamin D deficiency and secondary hyperparathyroidism in the elderly: consequences for bone loss and fractures and therapeutic implications. Endocrine Reviews 22(4): 477-501.

3. Holick MF, Binkley NC, Bischoff-Ferrari HA, Gordon CM, Hanley DA, et al. (2011) Evaluation, treatment, and prevention of vitamin D deficiency: an Endocrine Society clinical practice guideline. The Journal of clinical endocrinology and metabolism 96(7): 19111930

4. Sprague S, Petrisor B, Scott T, Devji T, Phillips M, et al. (2015) What is the role of vitamin D supplementation in acute fracture patients? A systematic review and meta-analysis of the prevalence of hypovitaminosis D and supplementation efficacy. Journal of orthopaedic trauma 30(2): 53-63.

5. American Geriatrics Society Workgroup on Vitamin D Supplementation for Older Adults (2014) Recommendations abstracted from the American Geriatrics Society Consensus Statement on vitamin D for Prevention of Falls and Their Consequences. J Am Geriatr Soc 62(1): 147-152.

6. Van Schoor NM, Lips P (2011) Worldwide vitamin D status. Best practice \& research Clinical endocrinology \& metabolism 25(4): 671-680.

7. Whiting SJ, Calvo MS (2005) Dietary recommendations for vitamin D: a critical need for functional end points to establish an estimated average requirement. J Nutr 135(2): 304-309.

8. Soontrapa S, Soontrapa S, Chailurkit L, Khampitak T (2002) The prevalence and the calcidiol levels of vitamin $\mathrm{D}$ deficiency in the elderly Thai women in municipality of Khon Kaen province, Thailand. Srinagarind Med J 17(4): 231-238.

9. Chailurkit L, Aekplakorn W, Ongphiphadhanakul B (2011) Regional variation and determinants of vitamin D status in sunshine-abundant Thailand: BMC Public Health 11: 853.

10. Vogeser M, Kyruatsoulis A, Huber E, Kobold U (2004) Candidate Reference method for the Quantification of Circulation 25-Hydroxyvitamin D3 by liquid Chomatography-Tandem Mass Spectrometry. Clin Chem 50(8): 1415-1417.

11. Charlson ME, Pompei P, Ales KL, Mackenzie CR (1987) A new method of classifying prognostic co morbidity in longitudinal studies: development and validation. J Chronic Dis 40(5): 373-383.

12. Bogunovic L, Kim AD, Beamer BS, Nguyen J, Lane JM (2010) Hypovitaminosis D in patients scheduled to undergo orthopaedic surgery: a single-center analysis. The Journal of Bone and Joint Surgery (American volume) 92(13): 2300-2304. 


\section{Journal of Orthopedics \& Bone Disorders}

13. Lips $P$ (2007) Vitamin D status and nutrition in Europe and Asia. The Journal of Steroid Biochemistry and Molecular Biology 103(3-5): 620-625.

14. Michelson JD, Charlson MD (2016) Vitamin D Status in an Elective Orthopedic Surgical Population. Foot \& ankle international 37(2): 186-191.

15. Gumieiro DN, Murino Rafacho BP, Buzati Pereira BL, Cavallari KA, Tanni SE, et al. (2015) Vitamin D serum levels are associated with handgrip strength but not with muscle mass or length of hospital stay after hip fracture. Nutrition (Burbank, Los Angeles County, Calif) 31(7-8): 931-934.

16. Maier GS, Maus U, Lazovic D, Horas K, Roth KE, et al. (2016) Is there an association between low serum 25OH-D levels and the length of hospital stay in orthopaedic patients after arthroplasty? Journal of orthopaedics and traumatology: official journal of the Italian Society of Orthopaedics and Traumatology 17(4): 297-302. 